\title{
High-fidelity tomographic imaging reconstruction for photopyroelectric thermal wave detection equipment
}

\author{
A. Yarai, K. Sakamoto and T. Nakanishi \\ Department of Electrical Engineering and Electronics, Faculty of Engineering, Osaka Sangyo University, \\ Daito, Osaka, Japan
}

\begin{abstract}
This paper describes a high-fidelity tomographic imaging reconstruction method for photopyroelectric thermal wave detection equipment. The high-fidelity is achieved through a high signal-to-noise ratio detection technique and through a new calculation algorithm. A very-smalldiameter detection metal probe tip can be used due to the high signal-to-noise ratio detection technique, increasing the spatial resolution. The calculation algorithm which considers thermal wave transmission paths along with defects achieves the high-fidelity tomographic imaging reconstruction.
\end{abstract}

\section{INTRODUCTION}

A new photopyroelectric tomographic imaging technique has recently been developed[1]-[3]. This technique adopts a thermal wave detection method, whose key components are a photopyroelectric film having an electrode on one side and a capacitive coupled remote metal tip (i.e. noncontacting) capable of sampling the local thermal wave field value. Therefore, this technique is effective in non-destructively evaluating the sub-surface features of solids. It has, however, serious difficulty with high-fidelity tomographic imaging reconstruction due to a very low signal-to-noise ratio (very low detected signal amplitude, on the order of $\mathrm{nV} \sim 10 \mathrm{nV}$ ). Moreover, a serious problem exists with position discrepancies of reconstructed sub-surface defects from the real positions.

This paper shows that high-fidelity tomographic imaging reconstruction is possible with (1) a high signal-to-noise ratio and high spatial resolution, and (2) a new calculation algorithm. Note that (1) is achieved with (a) a film of high pyroelectric coefficient, (b) a very-high-power laser diode, (c) a very-smalldiameter metal probe tip and (d) ultra-low-noise pre- and 2nd-stage amplifiers. Note that (2) basically uses the one-dimensional heat-flow equation which considers thermal wave transmission paths along with defects.

\section{EXPERIMENTAL SETUP}

Figure 1 shows the experimental setup for the tomographic scans (both the excitation laser and the probe tip are scanned independently). In order to avoid thermal perturbation while the probe tip is being scanned, a gap is set between the probe tip and the pyroelectric film. By attaching an ultra-low-noise FET amplifier directly to the probe tip, a very low input capacitance of $5 \mathrm{pF}$ is obtained, suppressing a decrease in the signal voltage[5]. It should be noted that in our equipment (i.e. capacitive coupled pyroelectric detector), an $85-\mu \mathrm{m}$-thick $\mathrm{LiTaO}_{3}$ (Hamamatsu Photonics Co., Ltd.) pyroelectric film, which has a five-fold larger figure of merit than a 30- $\mu \mathrm{m}$-thick PVDF (polyvinylidene fluoride; KF piezo film, Kureha Chemical Industry Co., Ltd.) film, and a high power laser diode (wavelength: $840 \mathrm{~nm}$, power: $970 \mathrm{~mW}$ ) as an excitation heating source can be used. The beam is focused to an approximately $20-\mu \mathrm{m}$-diameter spot on the sample. Consequently, a signal-to-noise ratio of at least 100-times that in previous works [3][4] can be expected, as well as a small-sized experimental setup. 
When an $\mathrm{LiTaO}_{3}$ pyroelectric film was used, however, it was found that not enough measurement reproducibility could be obtained, probably due to the influence of humidity[6]. Therefore, it was necessary to control the humidity very carefully when the $\mathrm{LiTaO}_{3}$ film was used. This problem has not yet been completely resolved.

\section{TOMOGRAPHIC MEASUREMENT}

The measurement sample used was a $2.0-\mathrm{mm}$-thick and $15-\mathrm{mm}$-square aluminum plate with a $1.0-\mathrm{mm}$ diameter drilled out hole at its center (between the top and bottom surfaces) and with a thin layer of black paint on the top surface. The gap mentioned above was set at $0.1 \mathrm{~mm}$. The diameters of the metal (brass) probe tips were varied from $0.2 \mathrm{~mm} \sim 0.8 \mathrm{~mm}$. These parameters were selected quantitatively by finite element method (FEM) analysis[5]. The excitation beam was amplitude modulated in the frequency range of $10 \sim 100 \mathrm{~Hz}$ with a laser diode driver. Scan spans for the excitation laser and the probe tip scanned independently, were set at 4.0 $\mathrm{mm}$. Scan steps were $0.1 \mathrm{~mm}$.

\section{THERMAL WAVE BYPASS TRANSMISSION}

We calculated the bypass transmission effect of a thermal wave with the one-dimensional heat-flow equation. Figure 2 shows the theoretical and experimentally detected phase lag values. The calculation conditions were as follows. 1) a 0.4-mm-diameter metal probe tip was fixed at the bottom surface of the test sample, and set at the hole center. 2) The thermal wave transmission distance $\mathrm{x}$ was calculated; the flow was along the hole, between the laser irradiated position on the top surface and the metal probe tip position. In the figure, the origin is defined as that on the top surface, which corresponds to the point where the probe tip is fixed and the edge of the probe tip. 3) The phase lag value was calculated by using $x$. Here, the modulation frequency is $50 \mathrm{~Hz}$.

The figure shows that these experimental phase data closely match the calculated curve of a 1.6-mmdiameter probe tip, which is 4-fold that of a $0.4-\mathrm{mm}$-diameter probe tip. The reason for this is the fringing effect of electric field lines for the probe tip ${ }^{[5]}$. The correspondence between the theoretical (probe tip diameter is $1.6 \mathrm{~mm}$ ) and experimental values (probe tip diameter is $0.4 \mathrm{~mm}$ ) shows that the calculation condition whereby heat flow along the hole is proper (bypass transmission). A slight discrepancy, however, can yet be seen between the theoretical curve calculated with the one-dimensional heat-flow equation (probe tip diameter is $1.6 \mathrm{~mm}$ ) and the experimental data (probe tip diameter is $0.4 \mathrm{~mm}$ ). The reason for this is assumed that the one-dimensional heat-flow equation can not be adopted completely especially at the area adjacent to the hole boundary.

\section{TOMOGRAPHIC RECONSTRUCTION ALGORITHM}

As mentioned above, we have developed a new calculation algorithm which considers thermal wave transmission paths along with defects to be imaged. The one-dimensional heat-flow equation for this algorithm is solved as follows. (1) A cross-sectional area that includes a defect to be tomographically imaged is divided into picture pixel matrix. (2) The thermal transmission distance $\mathrm{x}$ is calculated between every laser and probe tip position along with the defect, by using the detected phase signals. Phase datum is used instead of amplitude datum because it is less susceptible to mechanical gap alignment errors and to configuration changes on the sample surface. (3) Values for $F(F=x / d)$ are calculated, where $d$ is the direct 
distance between the laser position and the probe tip position. An $F$ value represents the bypass transmission ratio of a thermal wave. (4) For every pixel, all $F$ values obtained using all phase data relating to the pixel (meaning data obtained when the direct path from a laser position to a probe tip crosses the pixel) are added. (5) Every pixel is classified into " 1 " or " 0 " according to the summed $F$ values (a value larger than a certain threshold level is classified as " 1 "). In this method, it is easy to find a reasonable threshold level.

A tomographic image reconstructed with this method, however, may suffer from image distortion and/or position errors. To overcome these problems, we extended the algorithm as follows. It was found that the errors occurred due to the ununiformity in the calculation (the adding mentioned above) times for every pixel. To correct this problem, each pixel was further divided 10 times (only for the top and bottom directions) and only for the calculation.

Figure 3 illustrates the adding times of locations nearby a center position for a sample with no defect before and after this additional division. With this new calculation algorithm, the image distortion and position errors, which had been serious problems, were significantly reduced.

\section{RECONSTRUCTED TOMOGRAPHIC IMAGE}

Owing to the techniques mentioned above, a very-smalldiameter probe tip of $0.2 \mathrm{~mm}$ diameter could be used, drastically increasing the spatial resolution. Figure 4 shows the modulation frequency dependence of the position deviation quantity (the positions deviate to the bottom direction), with and without the correction shown is the previous section. Figure 5 shows the modulation frequency dependence of the image fidelity factor, with and without the correction. As shown in the figure, the circle obtained had an almost complete shape, where the image fidelity factor was calculated to be $93 \%$ when the modulation frequency is $50 \mathrm{~Hz}$. Here, the image fidelity factor is defined to be the ratio of 1) the white area obtained from the real data within the original complete circle, over 2) the area of the original complete circle. It was found that this new algorithm considerably corrects both the position deviation quantity and the image distortion of defects [3]-[4].

Figures 6 shows a phase datum for a probe tip of 0.2 $\mathrm{mm}$, and Fig. 7 shows reconstructed tomographic images for a probe tip of $0.2 \mathrm{~mm}$ or $0.6 \mathrm{~mm}$, when the modulation frequency is $50 \mathrm{~Hz}$. It is shown that the spatial resolution of the former is well improved compared with that of the latter.

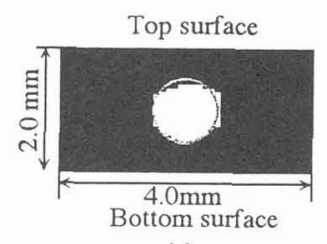

(a)

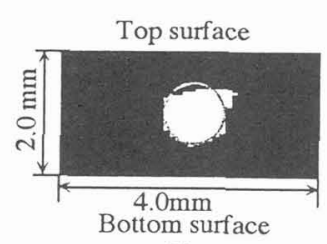

(b)

Fig. 7 Phase reconstruction images probe tip diameter: (a) $0.2 \mathrm{~mm}$

(b) $0.6 \mathrm{~mm}$

$$
\begin{array}{l|l|l|l|l}
68 & 59 & 59 & 59 & 68 \\
\hline 67 & 69 & 59 & 69 & 67 \\
\hline 67 & 69 & 81 & 69 & 67 \\
\hline 66 & 79 & 79 & 79 & 66 \\
\hline 77 & 77 & 77 & 77 & 77
\end{array}
$$

(a) conventional algorithm

\begin{tabular}{l|l|l|l|l}
65 & 72 & 72 & 72 & 65 \\
\hline 68 & 76 & 76 & 76 & 68 \\
\hline 69 & 72 & 81 & 72 & 69 \\
\hline 68 & 76 & 76 & 76 & 68 \\
\hline 65 & 72 & 72 & 72 & 65
\end{tabular}

(b) new algorithm

Fig. 3 Adding times of locations nearby a center position for a sample with no defect

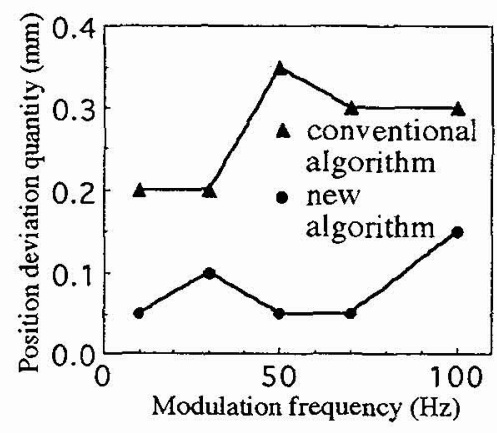

Fig. 4 Modulation frequency dependence of the position deviation quantity

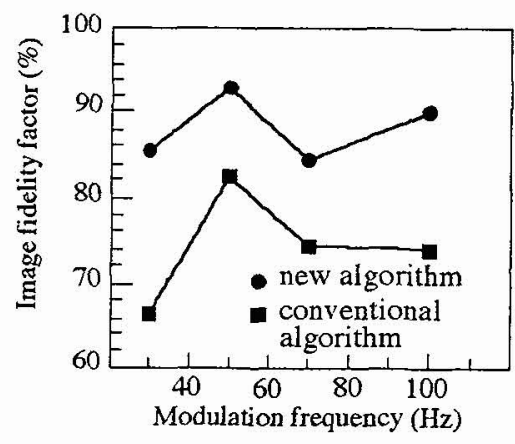

Fig. 5 Modulation frequency dependence of the image fidelity factor

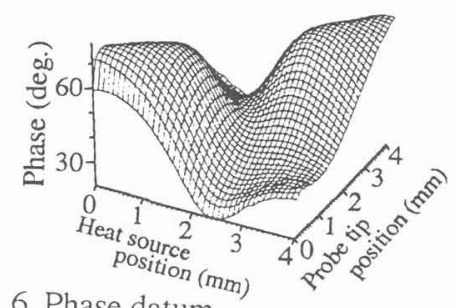

Fig. 6 Phase datum modulation frequency: $50 \mathrm{~Hz}$ probe tip diameter: $0.2 \mathrm{~mm}$ 


\section{CONCLUSION}

Owing to the high signal-to-noise ratio detection technique, high resolution detection has been achieved, drastically increasing the spatial resolution. We have developed a new reconstruction calculation algorithm which considers thermal wave transmission paths along with defects. Although this calculation algorithm is simple, a high-fidelity tomographic image could be obtained.

This work can be expected to yield significant tomographic reconstruction capabilities, even for thin solid materials with near-surface defects. An array of probe tips for parallel data collection is now being developed[7] to decrease the data collection time.

\section{References}

[1] M. Mieszkowski, K. F. Leung and A. Mandelis, Rev. Sci. Instrum. 60 (1989) 3.

[2] M. Mieszkowski and A. Mandelis, J. Opt. Soc. Am. A7 (1990) 552.

[3] M. Munidasa and A. Mandelis, J. Opt. Soc. Am. A8 (1991) 1852.

[4] M. Munidasa, A. Mandelis and C. Ferguson, Appl. Phys. A54 (1992) 244.

[5] A. Yarai, K. Sakamoto and T. Nakanishi, Jpn. J. Appl. Phys. 33 SB (1994), in printing.

[6] A. Yarai, K. Sakamoto and T. Nakanishi, FERROELECTRICS (1994), in printing.

[7] A. Yarai, Y. Yokoyama, K. Sakamoto and T. Nakanishi, Proc. IEEE IMTC '94, THP1-10, 1994. 